\title{
Las redes sociales como motivación para el aprendizaje: opinión de los adolescentes
}

\section{Social networks as motivation for learning: perception of adolescents}

\author{
Isabel Dans Álvarez de Sotomayor \\ Departamento de Didácticas Específicas, Universidad de Vigo, España (isabel.dans@udc.es) \\ Pablo César Muñoz Carril \\ Departamento de Didáctica y Organización Escolar. Universidad de Santiago de Compostela, España \\ (pablocesar.munoz@usc.es)
}

Recibido el 11 de noviembre de 2015; revisado el 12 de febrero de 2016; aceptado el 29 de febrero de 2016; publicado el 1 de junio de 2016

\section{RESUMEN:}

La sociedad del conocimiento pone en valor el uso de la tecnología como factor de transformación social, siendo el ámbito educativo uno de los contextos en los que la incorporación de las tecnologías de la información y de la comunicación (TIC) han tenido un gran calado. Bajo este prisma, en los últimos años el empleo extensivo de las redes sociales por parte del sector juvenil, así como su creciente incorporación en el ámbito escolar, han sido aspectos que no han pasado desapercibidos para el campo de la investigación. Precisamente, el presente artículo da a conocer los resultados obtenidos tras examinar la opinión de adolescentes estudiantes de $4^{\circ}$ ESO de A Coruña sobre las redes sociales como motivación para el aprendizaje. A través de algunas preguntas recogidas en un cuestionario más amplio (aplicado a 1.144 alumnos) se ha podido trazar la percepción del alumnado, como beneficiarios de la tecnología en la enseñanza, a fin de comprobar si favorece o no el aprendizaje escolar. Para ello se realizan análisis a través de estadística descriptiva y no paramétrica utilizando variables independientes como el género, tipo de centro, edad y las calificaciones del alumnado. Las conclusiones revelan que el uso de las redes sociales en las asignaturas las haría más atractivas. Asimismo, se han detectado diferencias por género, siendo las alumnas quienes consideran en mayor medida que las redes sociales son recursos que permiten la adquisición de nuevos aprendizajes. Según el tipo de centro y edad, son los estudiantes de centros concertados y de 15 años los que consideran que un uso prolongado de estas herramientas podrían afectar a su rendimiento académico. Finalmente, no se han encontrado valores estadísticamente significativos en el contraste de los ítems del cuestionario referentes a las calificaciones del alumnado respecto a su motivación para el aprendizaje con redes sociales.

\section{PALABRAS CLAVE: REDES SOCIALES, APRENDIZAJE, MOTIVACIÓN, ADOLESCENCIA, JUVENTUD.}

\begin{abstract}
:
The knowledge society enhances the use of technology as a factor of social transformation. The education sector being one of the contexts in which the incorporation of information technology and communication (ICT) has great significance. In light of this, in recent years the extensive use of social networks by the young and its increasing involvement in schools have been aspects that have not gone unnoticed in research. On this basis, this article seeks to highlight the results after considering the views of adolescent students in secondary education in the city of A Coruña on social networks as motivation for learning. A more comprehensive questionnaire (applied to 1,144 students) has allowed us to trace the opinions of users of technology on teaching themselves to check whether or not it is conducive to learning. This is done through descriptive analysis and non-parametric
\end{abstract}


statistics using dependent variables such as gender, type of centre, age, and qualifications of students. The findings reveal that the use of social networks when teaching subjects would make them more attractive. Gender differences have also been identified with the students who believe that social networks are resources that allow the acquisition of new learning. The study reveals that 15 year-old students in private schools are those who believe that prolonged use of these tools could improve their academic performance. Finally, they found no statistically significant values on contrasting variables concerning qualifications and motivation for learning with social networks.

\section{KEYWORDS: SOCIAL NETWORKS, LEARNING, MOTIVATION, ADOLESCENCE,} YOUTH.

\section{INTRODUCCIÓN}

La sociedad del conocimiento pone en valor el uso de la tecnología como factor de transformación social, tal como se recoge en la Cumbre Mundial sobre la Sociedad de la Información (Declaración de Principios y el Plan de Acción de la CMSI, que se aprobaron el 12 de diciembre de 2003). La tecnología se ha hecho mediadora de una adaptación de las relaciones sociales, los cambios en el trabajo y en las organizaciones que no deja indiferente al ámbito educativo. Precisamente, la inclusión digital y las agendas digitales se proponen el reto de los objetivos de la Unión Europea para el 2020. Es una prioridad reforzar la investigación, el desarrollo tecnológico y la innovación, gracias al desarrollo la inversión en educación digital y formación.

El sistema educativo se encuentra en un momento de transición: la euforia por la tecnología como remedio de los problemas escolares (Pardo, 2008; Morozov, 2012; Marqués, 2013) ha sido sustituida por una demandada formación del profesorado (Barberá, 2008; Cabero, 2007; Area, 2008). A pesar de esto y de la literatura sobre los beneficios de la tecnología, al igual que ocurre con el método de proyecto, la etapa de educación secundaria es más reacia a las innovaciones. Como señala Isorna, Navia y Felpeto (2013, p. 165) en el paso de primaria a secundaria se da un "cambio de la metodología didáctica: en ESO tienen mayor peso las exposiciones del profesor, el libro de texto y el aprendizaje memorístico, predomina un sistema de trabajo donde el grupo realiza la misma actividad a la vez". La falta de esfuerzos concretos en el cambio educativo en ESO y el vaivén legislativo han relegado esta tarea al debate teórico, lejos de las aulas. Además, la necesaria implicación de las familias en la educación de sus hijas e hijos se puede ver reforzada por el uso de las redes sociales. Los padres cuyos centros educativos incluyen estas herramientas en su educación están más informados sobre el rendimiento académico y el desempeño de tareas y objetivos de enseñanza, por su participación en las mismas (Iong, 2015).

Tomando en consideración estos aspectos, el presente artículo se estructura en tres grandes apartados de los que se da cuenta, en primer lugar, del maridaje entre tecnología y aprendizaje, así como de los motivos que promueven las redes sociales en los procesos educativos. A continuación, se exponen los principales resultados derivados de una investigación de carácter cuantitativo llevada a cabo con estudiantes de $4^{\circ}$ de educación secundaria obligatoria de la ciudad de A Coruña (España). En dicho estudio se analiza la opinión de los adolescentes sobre las redes sociales como recurso motivacional para su aprendizaje. $\mathrm{Al}$ mismo tiempo, a través de algunas preguntas recogidas en un cuestionario más amplio, se señala la percepción de los propios usuarios y beneficiarios de la tecnología en la enseñanza, a fin de comprobar si favorece o no el aprendizaje escolar. Finalmente, se plantean una serie de reflexiones a modo de conclusión relacionadas con los hallazgos derivados del estudio presentado y la adopción de sistemas tecnológicos apoyados en redes sociales como complemento a situaciones de enseñanza y aprendizaje.

\section{TECNOLOGÍA Y APRENDIZAJE: UN BINOMIO POSIBLE}

A modo de anuncio profético, a comienzos de la década de los 90, el profesor Ferrés (1993) resaltaba la necesidad de usar los medios en las escuelas para llegar a todos. En opinión de este autor, en una sociedad donde la enseñanza es obligatoria para todos, debería haber una auténtica obsesión por la audiencia, por llegar a todos, por evitar el fracaso escolar, por ser eficaces sobre todo con aquellos que más dificultad tienen y, por tanto, la única manera es adecuarse a la sensibilidad del receptor y manejar adecuadamente sus emociones.

Prensky en su obra Enseñar a nativos digitales (2011) señala la disyunción actual en el proceso educativo: por un lado caminan los anhelos de los profesores por reconducir a un alumnado cada vez más disperso y, por otro, el desencanto y tedio de los estudiantes en una educación formal alejada de la realidad. ¿Es la tecnología un camino para recuperar 
la frustración de unos y otros? (Martín, Picos y Egido, 2010). Hay diferentes concepciones y amplios debates en torno a esta cuestión, siendo la conclusión más frecuente el pensar que la tecnología solo es una herramienta. El matiz que permite examinar con precisión la cuestión está en este adverbio: ¿solo? Si bien queda demostrada en la historia cómo distintas "herramientas" han acompañado los cambios de las sociedades, también lo es el caso de las tecnologías de la información y comunicación. En resumen, "las TIC no 'obran milagros' en el proceso de aprendizaje del alumnado. Pueden incrementar la motivación por lo novedoso de la metodología didáctica utilizada, pero al final, el trabajo y esfuerzo del alumnado debe estar presente" (Mifsud, 2010; p. 18).

Ahora bien, la existencia de numerosos recursos constituye una fuente de riqueza y de posibilidades educativas. A mayor disponibilidad mayor libertad de elección, lo que configurará un modelo educativo más afín a los intereses y demandas del centro, del profesorado y del alumnado elector. Castaño, Maiz, Palacio y Villarroel (2008) enumeran una serie de errores y recomendaciones: el tecnocentrismo y el infocentrismo como propuestas a abandonar; y la motivación, la selección y la programación intelectual con un diseño sencillo, compartido y creativo como los indicadores de éxito.

Junto a ello gana posiciones la cultura del juego, la llamada gamificación, que asocia el factor lúdico al aprendizaje. Si bien la tecnología contribuye a la aportación de distintas actividades, que refuerzan la motivación, la fijación de ideas, etc. Un estudio reciente impulsado por la Asociación Española de Distribuidores y Editores de Software de Entretenimiento (2012) indica que el avance de la gamificación es mayor, donde se señala una aceptación pedagógica del $75 \%$ de los profesores encuestados.

Las redes sociales, aplicadas en contextos educativos, tienen especial relación con el denominado trabajo colaborativo. Según García Sans (2008) este sería el intercambio y el desarrollo de conocimiento por parte de grupos reducidos de iguales orientados a la consecución de fines académicos. Así pues, las potencialidades de las redes sociales resultan plenamente útiles si se ponen al servicio del trabajo colaborativo. La herramienta social apoyaría los beneficios de este: incrementa la motivación, favorece mayores niveles de rendimiento académico (puesto que el aprendizaje individual y el grupal se retroalimentan); mejora la retención de lo aprendido; potencia el pensamiento crítico y multiplica la diversidad de los conocimientos y experiencias que se adquieren (Hernández, González y Muñoz, 2014).

La tecnología en la enseñanza, es decir, la dotación de equipos e inversión en medios materiales es una realidad en los centros españoles. Con mayor o menor fortuna puede decirse que el nivel de cobertura técnica es amplia (Area et al., 2014). Junto a esta se da la realidad de la formación del profesorado, quien ha de diseñar, orientar e impulsar los aprendizajes dentro y fuera del aula. Los beneficios que comporta la enseñanza con tecnología suelen situarse en varios campos de actuación (Cachia, 2008; Castañeda, González y Serrano, 2011; De Haro, 2011; Poore, 2013), facilitan el aprendizaje al alumnado, favoreciendo la inclusión social y la atención a la diversidad. Contribuyen a la alfabetización digital y al desarrollo personal. Mejoran la comunicación interpersonal de la comunidad educativa. Son fuente de motivación para docentes y alumnos en un entorno atractivo (Sunkel y Trucco, 2010). Amplían el acceso, búsqueda y selección de la información, así como del almacenamiento y procesamiento de información en entornos diversos. Fomentan un aprendizaje con sentido y significativo, capaz de desarrollar el pensamiento crítico. Generan nuevas posibilidades de orientación y tutorización, así como de trabajo autónomo, cooperativo y colaborativo. Contribuyen a una visión interdisciplinar, que potencia la creatividad y el aprendizaje aplicado. Estas son algunas de las posibles ventajas de la tecnología educativa, que si bien han de concretarse para el caso específico de las redes sociales, gran parte de estos beneficios pueden ser extensivos a este tipo de herramientas comunicativas.

Sobre estas cuestiones existe literatura abundante, centrada en muchas ocasiones en la descripción del uso de las redes sociales por los menores, los riesgos y hábitos de conducta (Colás, González y De Pablos, 2013; Fundación Pfizer, 2009; Livinsgtone y Haddon, 2011; Livingstone, Haddon, Vincent, Mascheroni y Ólafsson, 2014; Rial, Braña, Gómez y Varela; 2014; Schwartz, 2013). Otros autores se han centrado en la relación de la tecnología con la enseñanza (Hamid, Waycott, Kurnia y Chang, 2015), pero no tenemos excesivas fuentes de información acerca de los resultados del aprendizaje. Los datos hasta ahora recogidos, tal como demuestra el último estudio de la OCDE (2015), caminan en la línea de que la tecnología no supone una mejora del rendimiento escolar, si bien estos efectos se suelen atribuir al uso inadecuado de la metodología tradicional. Todos los estudiantes deberían estar bien formados en habilidades básicas, 
para que se haga efectiva su participación plena en la competencia digital. El mero hecho de que existan los instrumentos no asegura el rendimiento escolar.

Desde EURYDICE se señala que "los métodos didácticos innovadores, basados en un aprendizaje activo y experiencial, que pueden verse beneficiados por el uso de las TIC, contribuyen a una mayor implicación del alumnado y a la mejora de sus resultados. Tanto en primaria como en secundaria, la inmensa mayoría de los países europeos recomiendan o sugieren diversos modelos didácticos innovadores" (2011; p. 43). Entre los que menciona el aprendizaje por proyectos, el aprendizaje personalizado, la enseñanza individualizada y la investigación científica.

\subsection{La motivación en la enseñanza - aprendizaje con redes sociales}

La creación de talento interdependiente, capacita al profesorado para utilizar su iniciativa y creatividad en colaboración con otros para contribuir a la comunidad educativa y aún a la sociedad (Cardona, 2002). Las redes sociales, como las demás herramientas TIC, son fuente de motivación extrínseca (se ve y se da a conocer la tarea que se realiza), intrínseca (en el nivel psicológico de satisfacción) y contributiva (se pone al servicio de la sociedad).

Sobre los usos y gratificaciones se delimitan cinco aspectos diferenciados, de acuerdo con Rubin (1993): a) la conducta comunicativa generada por el uso de los media es motivada o dirigida hacia una meta o fin; b) la audiencia selecciona y usa las fuentes de información y los mensajes con el fin de satisfacer necesidades o deseos; c) influyen factores sociales y psicológicos en la conducta comunicativa; d) los medios compiten con otras formas de comunicación por la selección, la atención y el uso; e) la intermediación de otros individuos es más influyente que los medios y sus mensajes por sí solos. Así, en el uso de los medios hay motivaciones cognoscitivas, afectivas y de integración. Generar, inferir, agrupar y transferir conocimiento se une a los estímulos de recompensa escolar y de mejora de las relaciones personales.

En concreto, las motivaciones de integración en el profesorado pueden sorprender, pero la necesidad de reconocimiento profesional (Leung, 2013) y reunión junto al resto de docentes de su propio centro educativo o de otros hacen de la relación una fuerte identidad profesional. Asimismo, en el caso de los adolescentes, Colás et al. (2013; p. 21) señalan "la necesidad social que tienen los jóvenes de compartir experiencias y también de reconocimiento de su actividad ante los demás, estableciendo nuevas relaciones sociales". Dicha gratificación, que pasa por sentirse identificado y aun diferenciado de forma exclusiva, está vinculado a la dimensión emocional. Conseguir un puesto único dentro de un grupo y lograr seleccionar lo relevante son algunas de las competencias que desarrollan este aprendizaje social.

Butler (1995) anuncia cómo el uso de Internet afecta a los hábitos cotidianos de los usuarios, que existan o no las herramientas digitales en la vida escolar no es un hecho indiferente. Otro autor, Yoo (1996) señala seis dimensiones de gratificación: entretenimiento, información, transacciones generales, transacciones laborales, creación de vínculos interpersonales y mantenimiento de los vínculos preexistentes, en la línea de lo que se expone en este artículo.

El panorama de adolescentes de secundaria, generación en red o Millenials, muestra una juventud que aprende, piensa y crea comunidades de forma diferente que sus padres. La televisión educativa existe, pero su éxito es menor en relación al fenómeno masivo de Internet en la educación. La interactividad es diferente, ya que la televisión es jerárquica y centralizada. En cambio, el mando de las tecnologías de la información y la comunicación parece ser llevado por la juventud. La red les da poder y las redes sociales son su medio seguro. Las relaciones de igualdad aparentes imponen que se les escuche y fomentan la participación. Tapscott (2008) propone diez características que definen a los protagonistas del ambiente digital: fuerte independencia; apertura emocional; inclusión social; libre expresión y puntos de vista firmes; innovación; preocupación por madurar; investigan y cambian; interactividad, inmediatez y velocidad; sensibilidad; autenticación, confianza en el anonimato, accesibilidad, diversidad y ubicuidad.

Con todo ello, el profesorado debe tomar posiciones de cambio. La repetición del modus operandi no parece el camino, ya que los profesionales de la enseñanza han de tener su propia estrategia. Es interesante la visión de Mora, Signes y De Miguel (2015), que demuestran la necesidad del docente como comunity manager. Si bien las redes sociales permiten la libertad de expresión que no se produce en el contexto educativo tradicional. Esta característica puede ser explotada para enriquecer el proceso educativo y mejorar la calidad de su logro.

La comunicación educativa admite mejor los formatos digitales que los audiovisuales de una forma natural. No se da este proceso, sin embargo, sin reticencias y prejuicios ligados en ocasiones a la 
falta de dirección (los “paraqués”). Además, los contenidos digitales por Internet e incluso el diseño de las redes sociales, son especialmente valoradas para el entretenimiento o el ocio. En esta línea, estudios como los de Colas et al. (2013); Dans (2014); Rial, Gómez, Braña y Varela (2014); Mora, Signes y De Miguel (2015), entre otros, afirman que los estudiantes consideran las redes sociales especialmente rentables desde el punto de vista educativo, pero su actividad fundamental es el ocio. El alumnado manifiesta atracción por las redes sociales como elemento de motivación hacia las asignaturas aunque, paradójicamente, aprecian las redes sociales como perturbadoras. Es necesario pues, el diseño y la programación educativa de las mismas.

\section{MATERIAL Y MÉTODO}

En la investigación desarrollada se utiliza una metodología cuantitativa de carácter no experimental, basada en el método de encuesta (Cohen y Manion, 1990; McMillan y Schumacher, 2005). Para ello, se diseña un cuestionario realizado ad hoc. Para asegurar el cumplimiento de aspectos psicométricos básicos, el instrumento fue evaluado por un panel de expertos especializados en metodología de investigación y tecnología educativa. También se realizó una prueba piloto, tras la cual se readaptaron y suprimieron diversos ítems del cuestionario a fin de mejorar su validez de contenido y de constructo. Asimismo, la fiabilidad obtenida de todo el cuestionario, mediante el índice de consistencia interna alfa de Cronbach, que arrojó un resultado de .937. Asimismo, es preciso señalar que para los ítems que forman parte de la dimensión analizada en el presente artículo, el nivel de fiabilidad ha sido de .749.

En relación a los participantes del estudio, se encuestaron a un total de 1.144 estudiantes de $4^{\circ}$ curso de enseñanza secundaria obligatoria de la ciudad de A Coruña (España), pertenecientes a 29 centros, 13 de carácter público, 15 privados y 1 concertado. En cuanto al género, el 47,2\% (n= 540) eran hombres y el 52,8\% (n=604) mujeres. En base a la edad, el 41,8\% $(n=478)$ eran estudiantes de 15 años, el $45,2 \% \quad(\mathrm{n}=517)$ de 16 años, el $10,6 \%$ $(\mathrm{n}=121)$ de 17 años $\mathrm{y}$, finalmente, el 2,4\% $(\mathrm{n}=28)$ de 18 años.

Según su distribución por tipología de centro, cabe señalar que 416 de los encuestados eran estudiantes de centros públicos, 689 de centros concertados y 39 de instituciones privadas.
El estudio pretende examinar la opinión de adolescentes estudiantes de $4^{\circ}$ ESO de la ciudad de A Coruña sobre las redes sociales como motivación para el aprendizaje, analizando la percepción de los propios usuarios y beneficiarios de la tecnología en la enseñanza, en concreto de las redes sociales, a fin de comprobar si favorece o no el aprendizaje escolar como elemento motivacional.

Con el fin de dar respuesta a los objetivos planteados en la investigación, los análisis desarrollados se articulan a través de estadística descriptiva y no paramétrica.

Mediante los análisis descriptivos se ha pretendido recoger, describir, organizar y sintetizar los datos observados, prestando especial atención a dos aspectos básicos como son: la tendencia central y la dispersión (Gil, 2000; Vilà y Bisquerra, 2004).

Respecto a los análisis no paramétricos, el propósito ha sido identificar diferencias significativas entre las variables objeto de estudio. Para ello se han utilizado análisis bivariados empleando la prueba U Mann Whitney para variables dicotómicas y Kruskal-Wallis para variables politómicas. En dichos análisis se han utilizado como variables independientes: el género, tipo de centro (público, privado y concertado), edad $(15,16,17$ y 18 años) y las calificaciones del alumnado (donde se han establecido dos categorías: "todas las asignaturas aprobadas" y "alguna asignatura suspensa"). Las variables dependientes utilizadas han sido: "El uso de las redes sociales en las asignaturas las haría más atractivas"; "En las redes sociales aprendo cosas diferentes"; "Las redes sociales contribuyen a la unidad y al sentimiento de equipo en una clase"; "Usar mucho las redes sociales afecta negativamente al estudio"; "Mis profesores no saben sobre redes sociales"; "Los profesores deberían utilizar las redes sociales en sus clases". A continuación se muestran los resultados obtenidos.

Hay que señalar que la utilización de pruebas no paramétricas obedece a que las distribuciones no siguen los principios de normalidad y homocedasticidad. 


\section{RESULTADOS}

\subsection{Opinión del alumnado de $4^{\circ}$ de ESO sobre el uso de las redes sociales como motivación para el aprendizaje}

En la tabla 1 puede verse cómo el resultado con mayor número de adhesiones es el que sugiere que el uso de las redes sociales harían más atractivas las asignaturas (puntuación media de 3,34). En este sentido parece claro que el alumnado reconoce las plataformas virtuales como motivadoras en sí mismas. Junto a este le sucede la opinión de que "en las redes sociales aprendo cosas diferentes" (promedio de 3,30). Tal diferencia parece guardar relación con la novedad y lo original como fuente de inspiración, junto con otros posibles contenidos no curriculares. Llama la atención que obtiene la misma puntuación media $(3,30)$ la opinión que vincula claramente las redes sociales al ocio, ya que opinan que su abuso afecta negativamente al estudio. Finalmente el aspecto emocional que genera unidad en la clase (puntuación media de 2,92), es decir, que favorece la comunicación, junto al hecho de ser recomendable su uso por parte del profesorado (promedio de 2,76), a quien una media de 2,68 acusan de desconocer el mundo de las redes sociales.

Tabla 1. Estadísticos descriptivos.

\begin{tabular}{ccccc}
\hline & $\mathbf{N}$ & Media & Desv. Típ. & $\begin{array}{c}\text { Error típico de } \\
\text { la media }\end{array}$ \\
El uso de las redes sociales en las asignaturas las haría más atractivas & 1144 & 3,34 & 1,374 & 0,41 \\
En las redes sociales aprendo cosas diferentes & 1144 & 3,30 & 1,265 & 0,37 \\
Las redes sociales contribuyen a la unidad y al sentimiento de equipo & 1144 & 2,92 & 1,246 & 0,37 \\
en una clase & 1144 & 3,30 & 1,416 & 0,42 \\
Usar mucho las RR.SS afecta negativamente al estudio & 1144 & 2,68 & 1,205 & 0,36 \\
Mis profesores no saben sobre redes sociales & 1144 & 2,76 & 1,338 & 0,40 \\
Los profesores deberían utilizar las RR.SS en sus clases &
\end{tabular}

\subsection{Diferencias de opinión del alumnado de $4^{\circ}$ de ESO por género, tipo de centro, edad y calificaciones sobre el uso de las redes sociales como motivación para el aprendizaje}

Tal y como se observa en la tabla 2 , se han encontrado diferencias según el género. En concreto, en las variables: "El uso de las Redes Sociales en las asignaturas las haría más atractivas" y "En las Redes Sociales aprendo cosas diferentes". En ambos casos, y a tenor de los rangos promedio obtenidos, son las alumnas las que están más de acuerdo con estos aspectos.

Tabla 2. Prueba U Mann-Whitney (variable de agrupación: género).

\begin{tabular}{|c|c|c|c|c|c|c|}
\hline & Género & $\mathrm{N}$ & Rango promedio & $\begin{array}{l}\text { Suma de } \\
\text { rangos }\end{array}$ & Estadísticos de & ontraste \\
\hline \multirow{4}{*}{$\begin{array}{c}\text { El uso de las redes sociales en } \\
\text { las asignaturas las haría más } \\
\text { atractivas }\end{array}$} & Hombre & 540 & 535,29 & 289057,00 & U de Mann-Whitney & 142987,000 \\
\hline & Mujer & 600 & 602,19 & 361313,00 & W de Wilcoxon & 289057,000 \\
\hline & Total & 1140 & & & $\mathrm{Z}$ & $-3,521$ \\
\hline & & & & & Sig. Asintótica &, 000 \\
\hline \multirow{4}{*}{$\begin{array}{l}\text { En las redes sociales aprendo } \\
\text { cosas diferentes }\end{array}$} & Hombre & 540 & 545,95 & 294812,50 & U de Mann-Whitney & 148742,500 \\
\hline & Mujer & 600 & 592,60 & 355557,50 & W de Wilcoxon & 294812,500 \\
\hline & Total & 1140 & & & Z & $-2,477$ \\
\hline & & & & & Sig. Asintótica & 013 \\
\hline \multirow{4}{*}{$\begin{array}{l}\text { Las redes sociales contribuyen } \\
\text { a la unidad y al } \\
\text { sentimiento de equipo en una } \\
\text { clase }\end{array}$} & Hombre & 540 & 551,30 & 297701,50 & U de Mann-Whitney & 151631,500 \\
\hline & Mujer & 600 & 587,78 & 352668,50 & W de Wilcoxon & 297701,500 \\
\hline & Total & 1140 & & & $\mathrm{Z}$ & $-1,935$ \\
\hline & & & & & Sig. Asintótica & ,053 \\
\hline \multirow{4}{*}{$\begin{array}{l}\text { Usar mucho las redes sociales } \\
\text { afecta negativamente al estudio }\end{array}$} & Hombre & 540 & 558,43 & 301550,00 & U de Mann-Whitney & 155480,000 \\
\hline & Mujer & 600 & 581,37 & 348820,00 & W de Wilcoxon & 301550,000 \\
\hline & Total & 1140 & & & $\mathrm{Z}$ & $-1,205$ \\
\hline & & & & & Sig. Asintótica & ,228 \\
\hline \multirow{2}{*}{$\begin{array}{l}\text { Mis profesores no saben sobre } \\
\text { redes sociales }\end{array}$} & Hombre & 540 & 588,63 & 317862,50 & U de Mann-Whitney & 152207,500 \\
\hline & Mujer & 600 & 554,18 & 332507,50 & W de Wilcoxon & 332507,500 \\
\hline
\end{tabular}




\begin{tabular}{|c|c|c|c|c|c|c|}
\hline & Género & $\mathrm{N}$ & Rango promedio & $\begin{array}{c}\text { Suma de } \\
\text { rangos }\end{array}$ & \multicolumn{2}{|c|}{ Estadísticos de contraste } \\
\hline \multirow{6}{*}{$\begin{array}{l}\text { Los profesores deberían utilizar } \\
\text { las redes sociales en sus clases }\end{array}$} & Total & 1140 & & & $\mathrm{Z}$ & $-1,842$ \\
\hline & & & & & Sig. Asintótica &, 065 \\
\hline & Hombre & 540 & 561,49 & 303207,00 & U de Mann-Whitney & 157137,000 \\
\hline & Mujer & 600 & 578,61 & 347163,00 & W de Wilcoxon & 303207,000 \\
\hline & Total & 1140 & & & $\mathrm{Z}$ &,- 901 \\
\hline & & & & & Sig. Asintótica & ,368 \\
\hline
\end{tabular}

Respecto al tipo de centro, y tras aplicar la prueba Kruskal-Wallis, tan solo se han hallado diferencias estadísticamente significativas en la variable "Usar mucho las redes sociales afecta negativamente al estudio" (p-valor =,013). En este caso, son los alumnos de centros concertados (rango promedio $=594,87$ ), frente a los públicos y privados (rangos promedio $=535,88$ y 567,91; respectivamente), los que opinan que un exceso de uso de las redes sociales podría tener repercusiones negativas en su rendimiento escolar.

En lo que atañe a la edad, cabe señalar que se han encuestado a alumnos con edades que oscilaban entre los 15 y los 18 años. Los resultados de la prueba Kruskal-Wallis evidencian que apenas existen diferencias entre los diversos grupos de edad. No obstante, en el contraste estadístico, sí se han encontrado valores inferiores a $\mathrm{p}=.05$ para la variable "Usar mucho las redes sociales afecta negativamente al estudio". Son los alumnos de 15 años los que están más de acuerdo con esta afirmación.

Por último, es preciso indicar que tras la aplicación de la prueba U Mann Whitney no se han encontrado valores estadísticamente significativos en el contraste de variables referentes a las calificaciones del alumnado (se han utilizado como categorías: "todas las materias aprobadas" y "alguna materia suspensa").

\section{DISCUSIÓN}

La motivación se ha hecho un elemento imprescindible en cualquier metodología de trabajo (Vadillo, 2013) y no es menor su impronta en el ámbito educativo (Peinado y Navarro, 2014). De hecho es una de las condiciones previas que garantizan la eficacia del uso de la tecnología en la enseñanza. Tras el fuerte impacto de las redes sociales en este primer cuarto de siglo, cabe señalar su influencia en la vida escolar (Almu y Alhaji, 2014; Barbour y Plough, 2012). De la seducción inicial por las herramientas virtuales hasta la necesaria volición y entrenamiento hay un largo camino que toca al profesorado recorrer junto a sus alumnos y alumnas. Son estos quienes hacen necesaria una revisión de las posturas extremas ante los medios en orden a los fines: la tecnofobia, que evita su uso Montero y Gewerc (2010), y la tecnofilia, que puede descuidar el aprendizaje (Cabero, 2007).

Los resultados aquí expuestos son fruto de una investigación más amplia que intenta dar voz a los propios jóvenes estudiantes. Al escuchar la voz de los interesados, verdaderos usuarios "autorizados" sin mediación alguna, también se logra hacerles partícipes del proceso educativo. La reflexión aporta un interesante feedback, pero es también construcción de la comunidad educativa, se trata de un aprendizaje social que interrelaciona los campos que están poco vinculados todavía (espacios digitales y presenciales). Sentirse reconocido a través del uso de las redes sociales por el desarrollo de otros aprendizajes y por el fomento de la unidad de la clase aparece como factor clave hacia las materias concretas. La práctica habitual que liga con frecuencia su utilización para el entretenimiento, fuera de la vida escolar, refuerza la idea de que las redes sociales afectan negativamente al estudio.

Así pues, se ha comprobado cómo el aserto que produce un mayor acuerdo es que el uso de las redes sociales en las asignaturas las haría más atractivas. Destaca la inclinación de las alumnas hacia esta afirmación, junto a la variable "En las Redes Sociales aprendo cosas diferentes". En cuanto a la edad no hay diferencias significativas, solo los alumnos de 15 años destacan que el uso excesivo de redes sociales puede afectar al estudio. Por centro educativo existe una tendencia de los estudiantes de centros concertados, quienes afirman el uso de las redes sociales como posible agente de dispersión frente al rendimiento académico. Si bien, esta cuestión puede referirse al uso habitual que los jóvenes hacen de dichos espacios digitales para el ocio, alejados de las otras tareas intelectuales o escolares. De hecho, el acuerdo sobre el uso de la herramienta como catalizador de la motivación escolar no guarda relación con el nivel de las calificaciones escolares, puesto que prácticamente no se detectan diferencias a pesar de sus expedientes académicos. 


\section{REFERENCIAS}

Agencia Ejecutiva en el ámbito Educativo, Audiovisual y Cultural (2011). Cifras clave sobre el uso de las TIC para el aprendizaje y la innovación en los centros escolares de Europa 2011. Instituto de Formación del Profesorado, Investigación e Innovación educativa. Secretaría general técnica: Bruselas.

Almu, A. y Alhaji, B. (2014). Effect of Mobile Social Networks on Secondary School Students. International Journal of Computer Science and Information Technologies, 5(5), 6333-6335. Recuperado de http://www.ijcsit.com/docs/Volume\%205/vol5issue05/ij csit2014050574.pdf.

Area, M. (2008). Las redes sociales en internet como espacios para la formación del profesorado. Razón y Palabra, 63. México. Recuperado http://www.razonypalabra.org.mx/n63/marea.html

Area, M., Alonso, C., , J., Correa, Moral, M., De Pablos, J., Paredes, J., Peirats, J. (2014). Las políticas educativas TIC en España después del Programa Escuela 2.0: las tendencias que emergen. Revista Latinoamericana de Tecnología Educativa. 13 (2), 11-33.

Asociación Española de Distribuidores y Editores de Software de Entretenimiento (2012). Estudio sobre el uso del videojuego en la enseñanza. Consultora GFK. Recuperado http://www.aevi.org.es/images/pdf/ppt_estudio.pdf.

Barberá, E. (2008). Calidad de la enseñanza 2.0. Revista de Educación a Distancia, VII. Recuperado de http://www.um.es/ead/red/M7/.

Barbour, M. K. y Plough, C. (2012). Odyssey of de Mind: Social Networking y CyberSchool. The International Review of Research in Open and Distance Learning, 13(3), 1-18. Recuperado de http://www.irrodl.org/index.php/irrodl/article/view/1154 $/ 2148$.

Butler, K. (1995). A uses and gratifications study of the internet. Master's Theses. Paper 1051. San Jose State University. Recuperado de http://goo.gl/1SnoU1

Cabero, J. (2007). Las necesidades de las TIC en el ámbito educativo: oportunidades, riesgos y necesidades. Tecnología y Comunicación Educativas., 45. Recuperado de http://investigacion.ilce.edu.mx/tyce/45/articulo1.pdf

Cachia, R. (2008). Los sitios de creación de redes. Aspectos sociales. Revista TELOS, 76. Recuperado de $\mathrm{http}: / /$ sociedadinformacion.fundacion.telefonica.com/tel os/articulocuaderno.asp@idarticulo=4yrev=76.htm

Cardona, P. (2002). Las claves del talento. Madrid: Empresa activa.

Castaño, C., Maiz, I; Palacio, G.; Villarroel, J. (2008). Prácticas educativas en entornos web 2.0. Madrid: Síntesis.

Castañeda, L.; González, V. y Serrano, J.L. (2011). Donde habitan los jóvenes: precisiones sobre un mundo de redes sociales. En Martínez, F. y Solano, I. Comunicación y relaciones sociales de los jóvenes en la red. Alicante: Marfil. pp. 47-63. Recuperado de http://digitum.um.es/jspui/bitstream/10201/25353/1/cast aneda_gonzalez_serrano.pdf

Cohen, L. y Manion, L. (1990). Métodos de investigación educativa. Madrid: La Muralla.

Colás, P., González, T. y J. de Pablos (2013). .Juventud y redes sociales: Motivaciones y usos preferentes. Comunicar. 20(40), 15-23. doi: http://dx.doi.org/10.3916/C40-201302-01
Cumbre Mundial sobre la Sociedad de la Información. Declaración de Principios y el Plan de Acción de la CMSI, que se aprobaron el 12 de diciembre de 2003. http://www.itu.int/wsis/index-es.html doi:10.1371/journal.pone.0073791http://www.plosone.o rg/article/info\%3Adoi\%2F10.1371\%2Fjournal.pone.007 3791.

Dans, I. (2014). Posibilidades educativas de las redes sociales. Tesis inédita. A Coruña: Universidade da Coruña.

De Haro, J. J. (2011). Redes sociales para la educación. Madrid: Anaya.

EURYDICE (2011). Cifras clave sobre el uso de las TIC para el aprendizaje y la innovación en los centros escolares de Europa 2011. Madrid: Ministerio de Educación.

Ferrés, J. (1993). La Educación audiovisual, asignatura pendiente de la reforma. Recuperado de http://www.lmi.ub.es/te/any93/ferres_cp2/. Fecha de visita: 15/10/2015.

Fundación Pfizer (2009). La Juventud y las redes sociales en Internet. Recuperado de https://www.fundacionpfizer.org/sites/default/files/pdf/I NFORME_FINAL_Encuesta_Juventud_y_Redes_Social es.pdf. Fecha de visita: 5/8/2013.

García Sans, A. (2008). Las redes sociales como herramientas para el aprendizaje colaborativo: una experiencia con Facebook. En Actas del XIII Congreso Internacional en Tecnologías para la Educación y el Conocimiento: la Web 2.0. Madrid: UNED.

Gil, J. A. (2000). Estadística e informática (SPSS) en la investigación descriptiva e inferencial. Madrid: UNED.

Hamid, S, Waycott, J. Kurnia, S. y Chang, S. (2015). Understanding students' perceptions of the benefits of online social networking use for teaching and learning. The Internet and Higher Education, 26, 1-9. doi: 10.1016/j.iheduc.2015.02.004

Hernández, N., González, M. y Muñoz, P.C. (2014). La planificación del aprendizaje colaborativo en entornos virtuales. Comunicar, 42, 25-33. doi: http://dx.doi.org/10.3916/C42-2014-02.

Iong, S. (2015). Extending social networking into the secondary education sector. British Journal of Educational Technology. En prensa. doi: 10.1111/bjet.12259

Isorna, M., Navia, C. y Felpeto M. (2013). La transición de la educación primaria a la educación secundaria. Innovación educativa, 23, 161-177.

Leung, L. (2013). Generational Differences in Content Generation in Social Media: The Roles of the Gratifications Sought and of Narcissism. Computers in Human Behavior, 29(3), 997-1006. doi:10.1016/j.chb.2012.12.028.

Livingstone, S. y Haddon, L. (2011). EU Kids Online: Final Report. LSE, London: EU Kids Online. Recuperado de www.eukidsonline.net.

Livingstone, S., Haddon, L., Vincent, J., Mascheroni, G. and Ólafsson, K. (2014). Net Children Go Mobile: The UK Report. London: London School of Economics and Political Science. Recuperado de: http://goo.gl/vfJwt0

Marquès, P. (2013). Investigación sobre el curriculum bimodal y contra el fracaso escolar. Recuperado de http://www.slideshare.net/peremarques/curriculumbimo dal investigacin-dimfundacin-telefnica.

Martín, A. G., Picos, A. P., y Egido, L. T. (2010). La formación de los futuros maestros y la integración de las TIC en la educación: anatomía de un desencuentro. Revista de Educación, 352, 215-231.

McMillan, J. y Schumacher, S. (2005). Investigación educativa. Madrid: Pearson Addison Wesley. 
Mifsud, E. (2010). Buenas prácticas TIC. Valencia: Generalitat Valenciana. Recuperado dehttp://www.cece.gva.es/dgite/docs/Buenas_Prac_Tic.p df

Mora, H., Signes, M. y De Miguel, M. (2015). Management of social networks in the educational process. Computers in Human Behavior, 12, 890-895. DOI: 10.1016/j.chb.2014.11.010

Montero, M. L. y Gewerc, A. (2010). De la innovación deseada a la innovación posible. Escuelas alteradas por las TIC. Revista de Curriculum y Formación del Profesorado, 14(1), 303-318.

Morozov, E. (2012). El desengaño de internet. Barcelona: Destino.

OCDE (2015). Students, Computers and Learning: Making the Connection. Paris: OCDE: doi: http://dx.doi.org/10.1787/9789264239555-en

Pardo, C. y Cobo, H. (2008). Planeta 2.0. Inteligencia colectiva o medios fast food. Barcelona /México DF: GRID-UVic; Flacso. Recuperado de: http://www.planetaweb2.net/

Peinado, P. y Navarro, D. (2014). Aumento de la motivación mediante el uso de redes sociales. Didáctica, innovación y multimedia, 29, 1-15.

Prensky, M. (2011). Enseñar a nativos digitales. Madrid: SM.

Poore, M. (2013). Using social media in the classroom. London: Sage.

Rial, A., Gómez, P., Braña, T. y Varela, J. (2014). Actitudes, percepciones y uso de Internet y las redes sociales entre los adolescentes de la comunidad gallega (España). Anales de Psicología, 30, 642-655. doi:10.6018/analesps.30.2.159111

Rubin, K. H., y Asendorpf, J. (1993). Social withdrawal, inhibition, and shyness in childhood: Conceptual and definitional issues. In K. H. Rubin y J. B. Asendorpf (Eds.), Social withdrawal, inhibition and shyness in children (pp. 3-17). Hillsdale, NJ: Erlbaum.

Schwartz H. A (2013). Personality, Gender, and Age in the Language of Social Media: The Open-Vocabulary Approach. PLoS ONE, 8(9). doi: http://journals.plos.org/plosone/article?id=10.1371/journ al.pone.0073791

Sunkel, G., y Trucco, D. (2010). TIC para la educación en América Latina. Riesgos y oportunidades. Serie Políticas Sociales, 167.

Tapscott, D. (2008). Grown Up Digital. How the Net Generation is Changing The World. New York: McGraw-Hill.

Vadillo, M. T. P. (2013). Liderazgo y motivación de equipos de trabajo. ESIC Editorial.

Vilà, R. y Bisquerra, R. (2004). El análisis cuantitativo de los datos. En Bisquerra, R. (coord.) Metodología de la investigación educativa (pp. 259-271). Madrid: Editorial la Muralla.

Yoo, H. (1996). Uses and gratifications of the Internet. Recuperado http://www.tc.msu.edu/tc960/yoofinal.htm 